\title{
Development of Stepping Motor Driver Based on DRV8825
}

\author{
Jian Huang \\ School of Xijing University, Xi'an 710123, China; \\ 565200245@qq.com
}

\begin{abstract}
In this paper, the working principle and the method of using DRV8825 to drive the stepper motor are described, and the hardware and software design are given. Based on the introduction of TI's DRV8825 stepper motor driver chip, the chip has 2.5A bipolar step on-chip 1/32 microstepping stepper motor indexer drive, especially suitable for driving small stepper motor. At present, it is widely used in 3D printers and micro stepping motors, and has certain practical value.
\end{abstract}

Keywords: Stepping motor; Microstepping; 3D printer

\section{基于DRV8825的步进电机驱动的研制}

\author{
黄健 \\ 西京学院, 陕西省西安市 710123, 中国
}

摘要: 本文阐述了利用DRV8825驱动步进电机的工作原理、使用方法并给出了具体的硬件和软件设计。在此基础上介绍 了德州仪器公司的步进电机驱动芯片DRV8825, 该芯片具有片上 $1 / 32$ 微步进分度器的 $2.5 \mathrm{~A}$ 双极步进电机驱动器, 特别适 合驱动小型步进电机。目前被广泛应用在3D打印机、微型步进电机上，具备一定的实用价值。

关键词: 步进电机; 微步进; 3D打印机

\section{引言:}

步进电机是将电脉冲信号转变为角位移或线位移的开环控制元, 可以分别通过控制脉冲个数和频率, 从而达到准确定位和调速的目的, 在机电一体化产品中有着广泛的应用。设计中常用的步进电机又有单极 型和双极型之分。相对而言, 单极型电机虽然应用效率较低, 但是驱动电路简单, 在早些年有较大的成本 优势, 特别是在高电压、大电流的应用中。不过近年来, 随着各大厂家双极型电机专用驱动芯片的大量推 出, 在性能不断提高的同时, 价格也在不断下降, 再综合了其占用 PCB 空间小, 控制简单等优点 ${ }^{[1-3]}$. 采用 双极型电机及专用驱动芯片取代单极性电机已经成为了一种趋势。本文将介绍一种双极型电机专用控制芯 片 DRV8825，并提供一个基于该芯片的打印机电机驱动电路设计方案。

\section{DRV8825 芯片介绍。}

DRV8825 的引脚图如图 1 所示。图中 STEP 引脚控制电机的步进, 每个上升沿电机步进 1 步, 内部带有 上拉电阻。DIR 控制电机的转动方向, DIR 为高电平, 电机顺时针旋转; DIR 为低电平, 电机逆时针旋转 [4]。 MODE0、MODE1、MODE2 控制电机的工作方式, 具体情况如表 1 所示。 


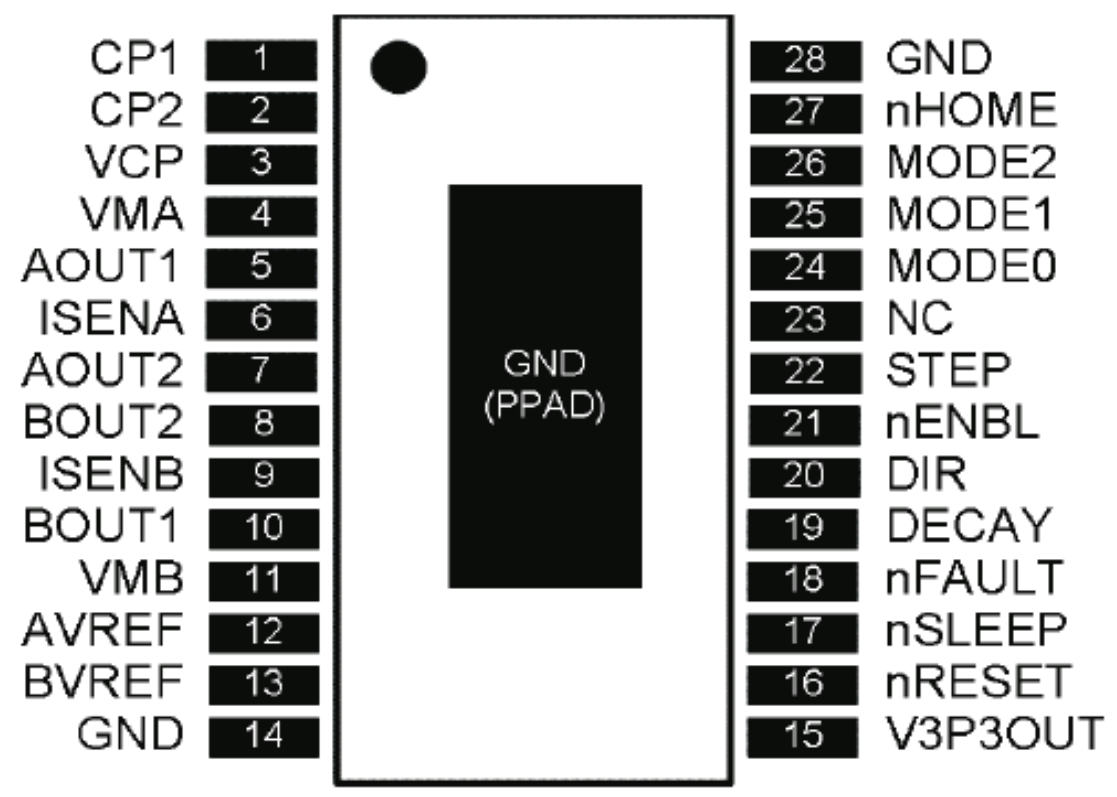

图 1 DRV8825 引脚图

表 1 步进电机的工作方式选择

\begin{tabular}{|c|c|c|c|}
\hline MODE0 & MODE1 & MODE2 & 步进的工作模式 \\
\hline 0 & 0 & 0 & 全步进 \\
\hline 0 & 0 & 1 & $1 / 2$ 步进 \\
\hline 0 & 1 & 0 & $1 / 4$ 步进 \\
\hline 0 & 1 & 1 & $1 / 8$ 步进 \\
\hline 1 & 0 & 0 & $1 / 16$ 步进 \\
\hline 1 & 0 & 1 & $1 / 32$ 步进 \\
\hline 1 & 1 & 0 & $1 / 32$ 步进 \\
\hline 1 & 1 & 1 & $1 / 32$ 步进 \\
\hline
\end{tabular}

根据表 1 可以选择步进电机的工作方式，当 MODE0、MODE1、MODE2 都为 0 时，是全步进工作方式; 当 MODE0、MODE1、MODE2 都为 1 时, 是 32 细分步进工作方式。

DRV8825 芯片还具有以下特性:

供电电压 $8.2 \mathrm{~V} \sim 42 \mathrm{~V}$.

最大电流 2.5A.

$3.3 \mathrm{~V}$ 的控制信号接口。

控制接口简单。

\section{DRV8825 步进电机驱动原理图。}

DRV8825 步进电机驱动的原理图如图 2 所示。图中左边是微处理器, 连接 STEP 的微处理器引脚要用定 时器产生 PWM 波, 电机转速的快慢与 PWM 波的频率有关。频率越快, 电机转速越快, 频率越慢, 电机转速 越慢。本设计中, 微处理器采用基于 Cortex-M3 的 STM32F103RBT6, 用其中的一个定时器 Timer1 产生 PWM 波。实验中, 步进电机采用 42 步进电机, 驱动时, PWM 波的频率范围从 $11 \mathrm{KHz}$ 到 $37 \mathrm{KHz}$, 速度从低到高, 在 $11 \mathrm{KHz}$ 是最慢, 但是驱动电流最大; 在 $37 \mathrm{~K}$ 时速度最快, 但是驱动电流最小, 图 3 中给出了详细的驱动 
电流图。其它引脚的连接, 参照前面介绍。

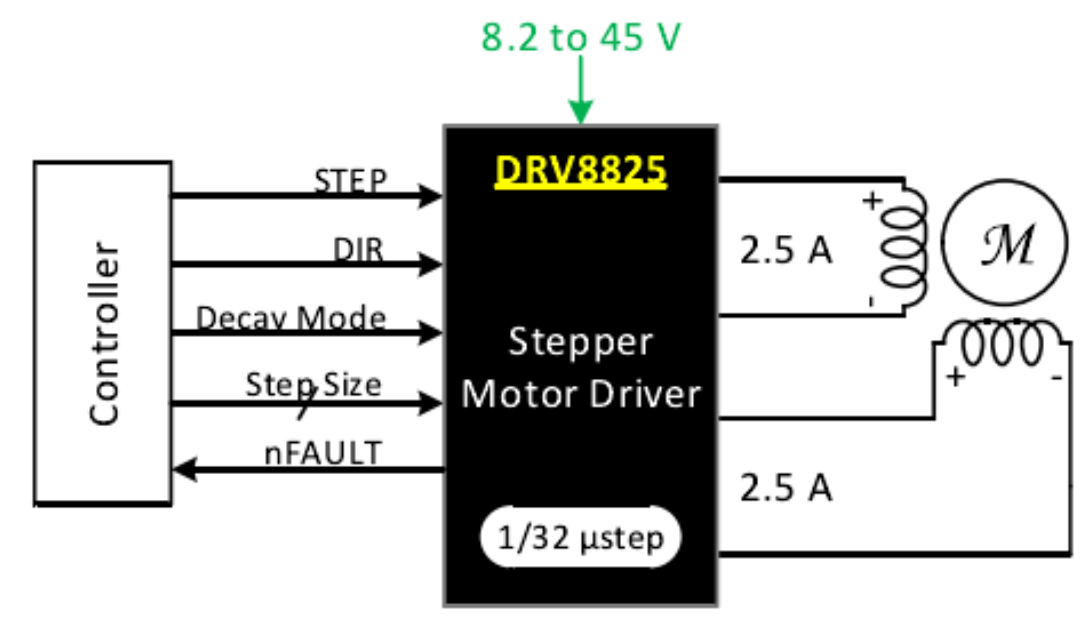

图 2. 步进电机驱动原理图

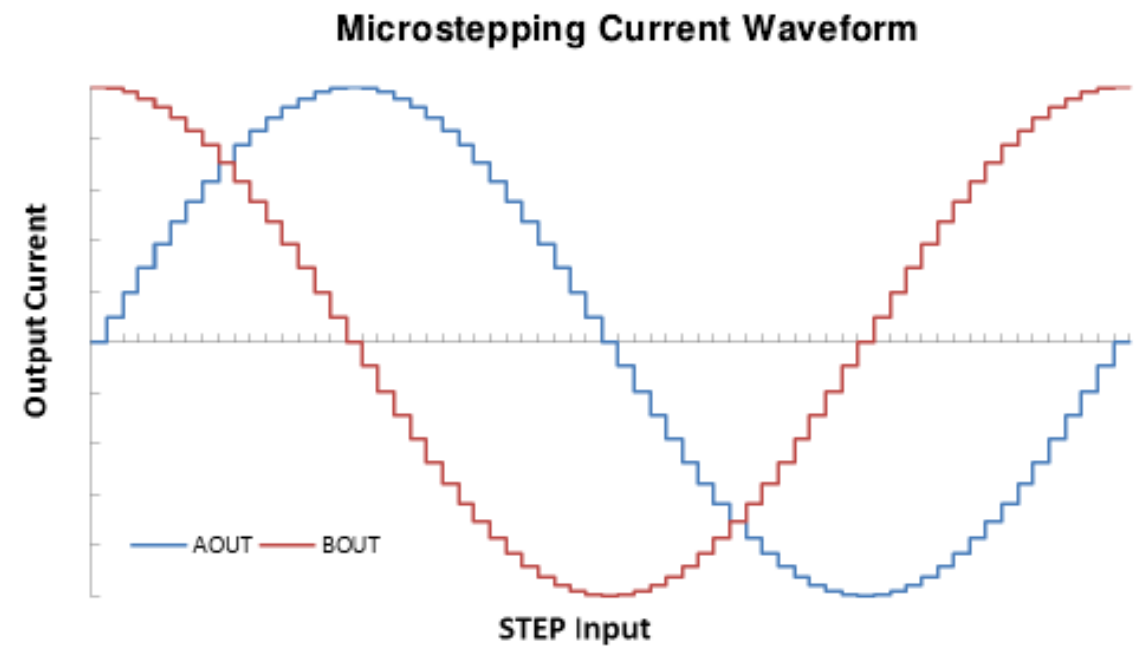

图 3. 驱动电流时序图

\section{3. 软件编程}

在本设计中, 在 KEIL 5 下用 C 语言编程。首先, 初始化定时器和电机, 然后用按键启动电机旋转, 并用按键调整 PWM 波的输出频率, 就可控制电机的转速。给出部分代码如下:

定时器初始化代码如下

Void Timer1_Init (u16 arr, u16 psc)

RCC- $>$ APB2ENR $\mid=1<<11$;

TIM1 $>$ ARR= arr;

TIM1->PSC=psc;

TIM1->DIER $\mid=1<<0$;

TIM1->DIER $\mid=1<<6$;

TIM1->CR1|=0x01;

MY_NVIC_Init(1,3,TIM1_UP_IRQn,2); 
按键调速代码如下:

Void EXTI1_IRQHandler (void)

Delay _ ms(10);

If $($ KEY $1==0)$

LED1=! LED1;

Key_flag=2;

TIM1_pwm-=180;

Printf ("IrınKey2 pwm =\%d", TIM1_pwm);

If (TIM1_pwm<=0) pwm _add=35000;

TIM1_PWM_Set (35999, 0, 3, 1, TIM1_pwm);

TIM1_PWM_Set (35999, 0, 3, 2, TIM1_pwm);

TIM1_PWM_Set (35999, 0, 3, 3, TIM1_pwm);

TIM1_PWM_Set (35999, 0, 3, 4, TIM1_pwm);

EXTI- $>$ PR $=1<<1$;

\section{4. 总结}

本文阐述了利用 DRV8825 驱动步进电机的工作原理和方法, 并给出了具体的应用, 实现了硬件电路和 软件编程。本设计具有广泛的应用价值, 可应用 3D 打印机、微电机控制等领域, 具有一定的实用价值。

\section{参考文献:}

[1] 吴迪. 基于 DRV8825DRV8825 的打印机电机驱动电路设计[J],电子技术与软件工程.2013, 6(27):62-63.

[2] 王昌喜, 杨先军 等. 基于三维加速度传感器的上肢动作识别系统[J],传感技术学报.2010, 23(6):816-819.

[3]高琴，刘淑聪. 步进电机控制系统的设计及应用[J]，制造业自动化.2012, 34(1):150-152.

[4] 王玉琳, 王强. 步进电机的速度调节方法[J],电机与控制应用.2006, 33(1):53-56.

\section{References:}

[1] Wu Di. Printer Motor Driving Circuit Design Based on DRV8825DRV8825 [J].Journal of Electronic Technology and Software Engineering, 2013, 6(27):62-63.

[2] Wang Changxi, Yang Xianjun, etc. Upper Action Recognition System Based on Triaxial Accelerometer [J].Chinese Journal of Sensors and Actuators, 2010, 23(6):816-819.

[3] Gao Qin, Liu Shucong. Design and Application of Control System of Stepping Motor [J].Manufacturing Automation, 2012, 34(1):150-152.

[4] Wang Yulin, Wang Qiang. Speed Regulation Methods of Stepping Motor [J].Electric Machines \& Control Application, 2006, 33(1):53-56. 